\title{
The value of combined detection of CEA, CYFRA21-1, SCC-Ag, and pro-GRP in the differential diagnosis of lung cancer
}

\author{
Jiezhou Li ${ }^{1}$, Yangqing Chen ${ }^{1}$, Xiandao Wang ${ }^{1}$, Chan Wang ${ }^{2}$, Meifang Xiao ${ }^{3}$ \\ ${ }^{1}$ Clinical Laboratory, Chengmai County People’s Hospital, Haikou, China; ${ }^{2}$ Emergency Department, Chengmai County People's Hospital, Haikou, \\ China; ${ }^{3}$ Clinical Laboratory, Hainan Women's and Children's Hospital, Haikou, China \\ Contributions: (I) Conception and design: J Li; (II) Administrative support: None; (III) Provision of study materials or patients: X Wang; (IV) \\ Collection and assembly of data: Y Chen, C Wang; (V) Data analysis and interpretation: M Xiao; (VI) Manuscript writing: All authors; (VII) Final \\ approval of manuscript: All authors. \\ Correspondence to: Meifang Xiao. Clinical Laboratory, Hainan Women’s and Children’s Hospital, Haikou, China. Email: sjianyanke@163.com.
}

Background: To investigate the value of carcinoembryonic antigen (CEA), cytokeratin 19 fragment (CYFRA21-1), squamous cell carcinoma antigen (SCC-Ag), and gastrin-releasing peptide (pro-GRP) in the differential diagnosis of lung cancer.

Methods: We enrolled 120 patients with malignant lung cancer who were treated at our hospital between June 2018 to June 2020. A further 58 patients with benign lung tumors and 60 healthy volunteers were also enrolled. Serum levels of CEA, CYFRA21-1, SCC-Ag, and pro-GRP were determined and compared across different populations, different pathological types, and different TNM stages. An ROC curve was drawn to evaluate the value of the four indicators when combined for the diagnosis of lung cancer.

Results: The levels of CEA, CYFRA21-1, SCC-Ag, and pro-GRP in the malignant group were significantly higher than those in the benign and healthy groups $(\mathrm{P}<0.05)$. CEA in adenocarcinoma was significantly higher than that in squamous cell carcinoma (SCC) and small cell carcinoma $(\mathrm{P}>0.05)$, and CYFRA21-1 in non-small cell carcinoma was significantly higher than that in small cell carcinoma $(\mathrm{P}<0.05)$. Pro-GRP in small cell carcinoma was significantly higher than that in non-small cell carcinoma $(\mathrm{P}<0.05)$, and the SCC-Ag level in SCC was significantly higher than that in small cell carcinoma and adenocarcinoma $(\mathrm{P}<0.05)$. There was no statistically significant difference in CEA among various pathological types $(\mathrm{P}>0.05)$. However, there were significant differences in the levels of CEA and pro-GRP in different TMN stages $(\mathrm{P}<0.05)$, and in the levels of CEA and pro-GRP in different TMN stages $(\mathrm{P}<0.05)$ where from stage I to stage IV CEA, pro -GRP levels increased. There was a significant difference in CYFRA21-1 levels in stages I-III $(\mathrm{P}<0.05)$, and in stages III and IV, although there was no statistically significant difference in SCC-Ag in different stages $(\mathrm{P}>0.05)$. The area under the curve (AUC) for the combined diagnosis of lung cancer with the four markers was 0.9250 (95\% CI: $0.8866-0.9634$ ), the sensitivity was $93.29 \%$, and the specificity was $84.32 \%$.

Conclusions: Joint inspection of CEA, CYFRA21-1, SCC-Ag, and pro-GRP levels has certain clinical value for the differential diagnosis of lung cancer.

Keywords: Lung cancer; carcinoembryonic antigen (CEA); CYFRA21-1; SCC-Ag; pro-GRP

Submitted Feb 03, 2021. Accepted for publication Apr 17, 2021.

doi: $10.21037 /$ tcr-21-527

View this article at: http://dx.doi.org/10.21037/tcr-21-527

\section{Introduction}

Lung cancer is the leading cause of cancer deaths in both men and women worldwide (1-3). Despite new diagnostic and genetic technologies, as well as many advances in surgical technology and biological treatments such as targeted therapy and immunotherapy, studies show that 
Table 1 Baseline data

\begin{tabular}{|c|c|c|c|}
\hline Project & Malignant group & Benign group & Healthy group \\
\hline Age & $50.84 \pm 20.76$ & $52.29 \pm 21.45$ & $49.43 \pm 20.51$ \\
\hline $\begin{array}{l}\text { Classification (NSCLC/squamous cell carcinoma/ } \\
\text { adenocarcinoma/Hamartoma/hemangioma) }\end{array}$ & 24/36/60/-/- & $-/-/-/ 36 / 22$ & - \\
\hline
\end{tabular}

the 5-year survival rate for lung cancer is only $15 \%$ (4). This is probably because most lung cancers are already at an advanced stage when diagnosed with only $15 \%$ of lung cancers diagnosed at an early stage, leading to a poor prognosis (5). Therefore, screening high-risk groups through low-dose computed tomography or effective biomarkers may facilitate the early diagnosis and early treatment of lung cancer and improve its prognosis (6-8).

Currently, biomarkers including carcinoembryonic antigen (CEA), cytokeratin 19 fragment (CYFRA21-1), squamous cell carcinoma antigen (SCC-Ag), and gastrinreleasing peptide (pro-GRP) have been found to be valuable in the diagnosis of lung cancer (9). CEA is a glycoprotein involved in cell adhesion which was previously considered a tumor marker for colorectal cancer, and SCC-Ag is a SCC-Ag which is both widely present in malignant epithelial cells and previously the first-choice tumor marker for cervical SCC (10). In recent years, with the continuous in-depth research on tumor markers, the application of CEA and SCC in lung cancer has also been reported (11). CYFRA21-1 is a fragment of cytokeratin 19 , which is currently considered to be a biomarker of non-small cell lung cancer (12). Pro-GRP is the precursor of gastrin releasing peptide (GRP) and both are tumor markers, but because GRP has a short half-life of only 2 minutes, the application of pro-GRP has become more common in recent years (13). Although they cannot replace histology in determining pathology, tumor markers play a significant role in the diagnosis of lung cancer, especially in clinical practice where determining the level of tumor markers is often the first step in screening. However, while the elevation of some serum markers can be observed in lung cancer cases and some are related to specific histopathological types, when used alone, their specificity and sensitivity are insufficient to meet the needs of diagnosis. For this reason, this study evaluated the roles of CEA, CYFRA21-1, SCC-Ag, and pro-GRP levels to assess their combined value in the differential diagnosis of lung cancer. We present the following article in accordance with the STARD reporting checklist (available at http://dx.doi. org/10.21037/tcr-21-527).

\section{Methods}

\section{Research object}

The malignant group consisted of 120 patients with lung cancer who were treated in our hospital between January 2017 and December 2020. The inclusion criteria were: (I) patients who had not received treatment; (II) patients who were diagnosed with lung cancer by pathological examination, and the pathological type and TNM staging had been confirmed; and (III) patients with complete clinical data and voluntary enrollment.

In addition, 58 patients diagnosed as having benign lung tumors by pathological examination were included in the benign group, and 60 healthy volunteers were included in the healthy group. The baseline information is shown in Table 1.

The study was conducted in accordance with the Declaration of Helsinki (as revised in 2013). The study was approved by Chengmai County People's Hospital (No.: 20161248). Written informed consent was obtained from the patients.

\section{Determination of CEA, CYFRA21-1, SCC-Ag, and pro- GRP levels}

We took $4 \mathrm{~mL}$ of fasting venous blood from all subjects, centrifuged it at 3,000 r/min for $10 \mathrm{~min}$, then measured CEA, CYFRA21-1, SCC-Ag, and pro-GRP levels by chemiluminescence method. The instrument was matched, and the measurement carried out in strict accordance with the instructions. The following reference ranges were used: CEA >5 ng/mL, SCC-Ag >1.5 ng/mL, CYFRA21-1 $>3.3 \mathrm{ng} / \mathrm{mL}$, and pro-GRP >65 pg/mL. 
Table 2 The levels of CEA, CYFRA21-1, SCC-Ag, and pro-GRP in different groups

\begin{tabular}{lccc}
\hline \multirow{2}{*}{ Biomarkers } & Small cell carcinoma $(\mathrm{n}=24)$ & \multicolumn{2}{c}{ Non-small cell lung cancer } \\
\cline { 3 - 4 } & & Squamous cell carcinoma $(\mathrm{n}=36)$ & Adenocarcinoma $(\mathrm{n}=60)$ \\
\hline CEA $(\mathrm{ng} / \mathrm{mL})$ & $8.74 \pm 4.47$ & $8.96 \pm 4.56$ & $10.43 \pm 3.88^{\mathrm{ab}}$ \\
CYFRA21-1 $(\mathrm{ng} / \mathrm{mL})$ & $6.84 \pm 2.91$ & $8.95 \pm 2.51^{\mathrm{a}}$ & $9.12 \pm 2.84^{\mathrm{a}}$ \\
SCC-Ag $(\mathrm{ng} / \mathrm{mL})$ & $5.23 \pm 2.79$ & $6.94 \pm 2.66^{\mathrm{a}}$ & $5.64 \pm 3.31^{\mathrm{b}}$ \\
pro-GRP $(\mathrm{pg} / \mathrm{mL})$ & $85.63 \pm 24.35^{\mathrm{bc}}$ & $65.48 \pm 26.23$ & $70.69 \pm 23.82$ \\
\hline
\end{tabular}

a, $\mathrm{P}<0.05$, compared with the small cell carcinoma group; ${ }^{\mathrm{b}}, \mathrm{P}<0.05$, compared with the squamous cell carcinoma group; ${ }^{\circ}, \mathrm{P}<0.05$, compared with the adenocarcinoma group. CEA, carcinoembryonic antigen; CYFRA21-1, cytokeratin 19 fragment; SCC-Ag, squamous cell carcinoma antigen; pro-GRP, gastrin-releasing peptide.

Table 3 The levels of four markers in different TMN stages

\begin{tabular}{|c|c|c|c|c|}
\hline Biomarkers & Phase I $(n=16)$ & Phase II $(n=35)$ & Phase III (n=42) & Phase IV $(n=27)$ \\
\hline CYFRA21-1 (ng/mL) & $6.64 \pm 2.11$ & $8.70 \pm 2.95^{\#}$ & $9.16 \pm 2.54^{\# \Delta}$ & $9.62 \pm 2.78$ \\
\hline SCC-Ag (ng/mL) & $3.46 \pm 1.72$ & $2.98 \pm 1.13$ & $3.08 \pm 1.03$ & $3.14 \pm 1.28$ \\
\hline pro-GRP (pg/mL) & $51.06 \pm 20.87$ & $65.06 \pm 23.72^{\#}$ & $76.38 \pm 24.36^{\# \Delta}$ & $89.42 \pm 25.62^{\# \Delta \Delta}$ \\
\hline
\end{tabular}

\#, P<0.05, compared with stage I; ${ }^{\Delta}, \mathrm{P}<0.05$, compared with stage II, ${ }^{\mathbf{\Delta}}, \mathrm{P}<0.05$, compared with stage III. CEA, carcinoembryonic antigen; CYFRA21-1, cytokeratin 19 fragment; SCC-Ag, squamous cell carcinoma antigen; pro-GRP, gastrin-releasing peptide.

\section{Statistical methods}

SPSS 20.0 software was used to perform statistical analysis on all experimental data. If the measurement data was expressed in the form of $x \pm s$, the independent sample $t$ test was used for comparison between the two groups. Diagnostic efficacy was evaluated by drawing the ROC curve.

\section{Results}

The levels of CEA, CYFRA21-1, SCC-Ag, and pro-GRP in different populations

The levels of CEA, CYFRA21-1, SCC-Ag, and pro-GRP in the malignant group were significantly higher than those in the benign and healthy groups $(\mathrm{P}<0.05)($ Table 2).

\section{The correlation between CEA, CYFRA21-1, SCC-Ag, pro- GRP, and TNM staging}

The levels of CEA and pro-GRP in different TMN stages were significantly different $(\mathrm{P}<0.05)$, and increased from stage I to stage IV. There was a significant difference in the level of CYFRA21-1 in stages I-III $(\mathrm{P}<0.05)$, and there was no significant difference between stages III and IV $(\mathrm{P}>0.05)$. There was no statistically significant difference in SCC-Ag in different stages $(\mathrm{P}>0.05)$ (Table 3).

\section{Diagnostic performance}

The area under the curve (AUC) for the combined diagnosis of lung cancer with the four markers was 0.9250 (95\% CI: $0.8866-0.9634$ ), the sensitivity was $93.29 \%$, and the specificity was $84.32 \%$ (Figure 1, Table 4).

\section{Discussion}

Biomarkers are biochemical indicators that change during the occurrence and development of disease. Tumor biomarkers exist in cancer tissues and host body fluids, and their levels can sometimes indicate the early-stage presence and nature of tumors, well before imaging and other assessment methods. However, all biomarkers are non-specific, and their sensitivity and specificity varies. Therefore, the combined use of multiple markers can improve diagnostic accuracy.

CEA is a common biomarker, the level of which is upregulated in a variety of malignant tumors. Although it has 

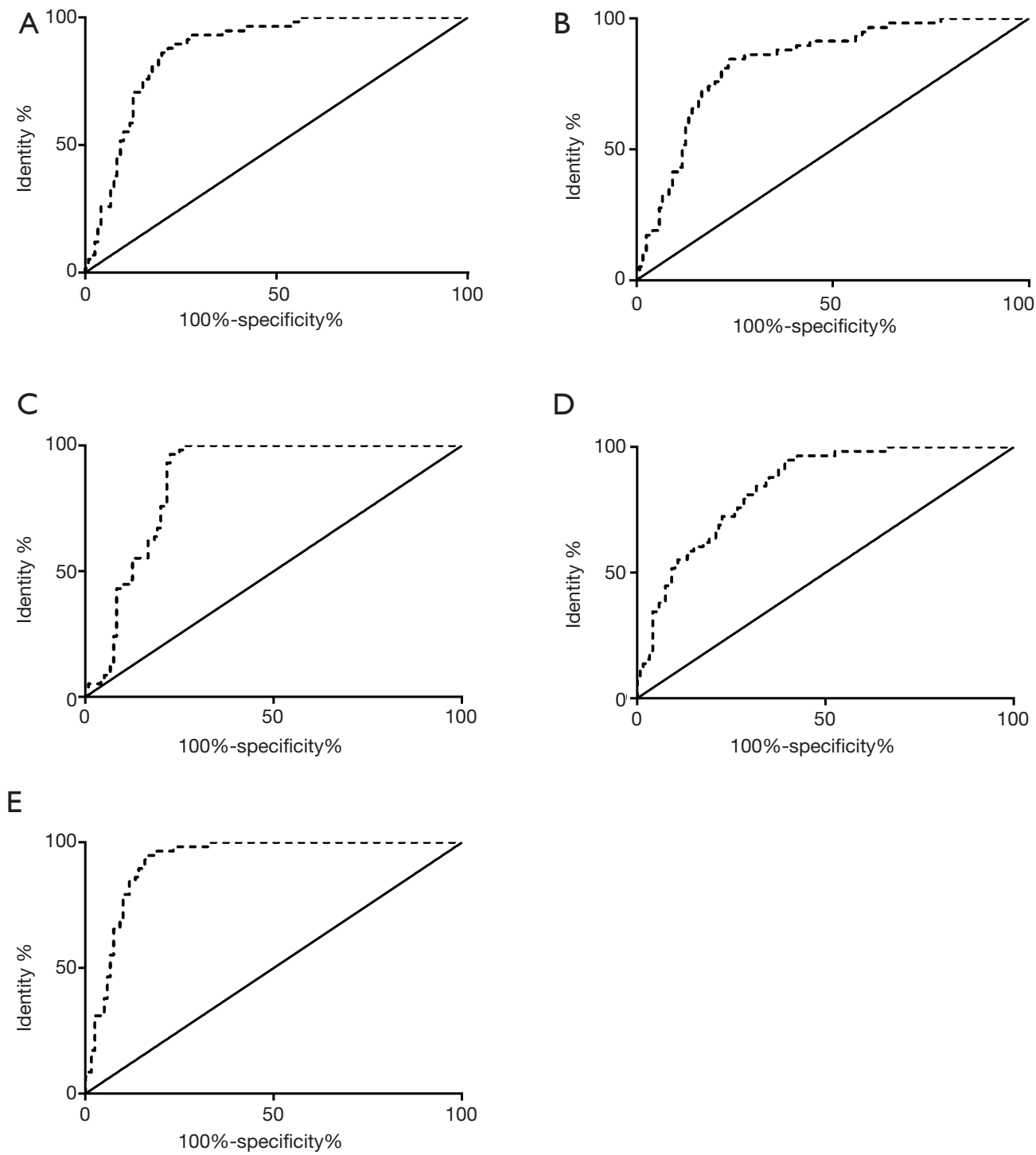

Figure 1 ROC curve of each marker. (A) CEA; (B) CYFRA21-1; (C) SCC-Ag; (D) pro-GRP; (E) joint. CEA, carcinoembryonic antigen; CYFRA21-1, cytokeratin 19 fragment; SCC-Ag, squamous cell carcinoma antigen; pro-GRP, gastrin-releasing peptide.

Table 4 AUC, sensitivity, and specificity of the four markers and combined diagnosis

\begin{tabular}{lcccc}
\hline Biomarkers & AUC & $95 \% \mathrm{Cl}$ & Sensitivity (\%) & Specificity (\%) \\
\hline CEA & 0.8739 & $0.8220-0.9251$ & 80.33 & 89.48 \\
CYFRA21-1 & 0.8332 & $0.7719-0.8948$ & 70.42 & 85.54 \\
SCC-Ag & 0.8642 & $0.8108-0.9176$ & 87.90 & 80.51 \\
pro-GRP & 0.8411 & $0.7843-0.8979$ & 85.85 & 79.20 \\
Four markers & 0.9250 & $0.8866-0.9634$ & 93.29 & 84.32 \\
\hline
\end{tabular}

CEA, carcinoembryonic antigen; CYFRA21-1, cytokeratin 19 fragment; SCC-Ag, squamous cell carcinoma antigen; pro-GRP, gastrinreleasing peptide. 
a broad spectrum, it still has value in the auxiliary diagnosis of lung cancer. As CEA is usually produced during fetal development, this stops before birth and CEA is usually not present in the blood of healthy adults. At present, CEA is more commonly applied to the diagnosis of adenocarcinoma in non-small cell lung cancer, but its cut-off level varies greatly in different studies $(14,15)$. In one Chinese study, the serum CEA level of non-small cell lung cancer cases was found to be significantly higher than that of benign lung tumor cases and healthy people (16), while in another, the level in a lung adenocarcinoma group was significantly higher than that of a lung SCC group and small cell lung cancer group (17). While results such as those mentioned above show that CEA is of great significance for the clinical diagnosis of lung cancer, its role in early diagnosis is less convincing as it has poor specificity and its level of increase in the early stage of lung cancer is not obvious. SCC$\mathrm{Ag}$ exists in squamous epithelial cells, participates in the differentiation of the squamous epithelial layer, participates in tumor growth in tumor cells, and is often used in the diagnosis of squamous epithelial cell-origin cancer (18). While initially used to screen for cervical cancer, later studies found that SCC-Ag levels are also elevated in lung SCC and SCC-Ag is an effective biomarker for that disease. Yu found that SCC-Ag levels in the serum of lung cancer patients was significantly higher than that of benign lung tumor cases and healthy people and $85 \%$ of patients with SCC-Ag levels greater than $2 \mathrm{ng} / \mathrm{mL}$ developed SCC (19). Serum SCC-Ag levels were significantly higher than that of lung adenocarcinoma cases, suggesting that serum SCC$\mathrm{Ag}$ has an important role in the detection of lung SCC. CYFRA21-1 is a characteristic marker of epithelial cells. When tumor cells die and dissolve, the soluble fragment of CK-19 CYFRA21-1 can be released into the blood, leading to an increase in the concentration of CYFRA21-1 in the serum of tumor patients. Although CYFRA21-1 is not an organ-specific and tumor-specific protein, it is particularly prone to appear at the junction of normal lung tissue and malignant tumors, making it an effective biomarker for lung cancer. Studies have shown that serum CYFRA21-1 is greater than $3.3 \mathrm{ng} / \mathrm{mL}$ in normal people and those with benign lung lesions, and the growth is more common in non-small cell lung cancer, with a sensitivity of about $50 \%$, and the sensitivity of SCC is higher (20). Pro-GRP is the precursor structure of GRP and is a relatively new lung cancer tumor marker. While researchers have found that GRP can exist in the neuroendocrine tissues of the brain, stomach, fetal lungs, and many small cell lung cancer cell lines and tumor groups, it is difficult to detect GRP in serum, so pro-GRP is usually measured. Cavalieri (21) showed that the blood pro-GRP level of patients with small cell lung cancer is higher than that of other types of lung cancer, and the positive rate and diagnostic specificity of pro-GRP in non-small cell lung cancer are low. However, pro-GRP has strong tissue specificity and other advantages compared with other tumor markers for small cell lung cancer such as NSE and Tu M-PK. Current research reports that the combined detection of multiple markers is of high value in the differential diagnosis of lung cancer $(22,23)$, but the combined detection of the four indicators of CEA, CYFRA21-1, SCC-Ag, and pro-GRP is rare.

The results of our study concur with those of others which indicate that CEA, CYFRA21-1, SCC-Ag, and proGRP play an important role in the differential diagnosis of lung cancer. The serum levels of CEA, CYFRA21-1, SCC-Ag, and pro-GRP in lung cancer cases were significantly higher than those in benign lung tumor cases and healthy people $(\mathrm{P}<0.05)$, indicating that they have certain significance in the diagnosis of lung cancer. Further exploration of the correlation of the four indicators with pathological types and TNM classification showed that CEA in adenocarcinoma was significantly higher than that in SCC and small cell carcinoma $(\mathrm{P}>0.05)$. CYFRA21-1 levels in non-small cell carcinoma were significantly higher than those in small cell carcinoma $(\mathrm{P}<0.05)$ and pro-GRP levels in small cell carcinoma were significantly higher than those in non-small cell carcinoma $(\mathrm{P}<0.05)$. In addition, the SCC-Ag level in SCC was significantly higher than seen in small cell carcinoma and adenocarcinoma $(\mathrm{P}<0.05)$. Across different TMN stages, the levels of CEA and proGRP were significantly different $(\mathrm{P}<0.05)$ and the levels of CEA and pro-GRP in stages I to IV increased. There was a significant difference in CYFRA21-1 levels in I-III $(\mathrm{P}<0.05)$, and no statistically significant difference between stages III and IV $(\mathrm{P}>0.05)$. The results show that CEA, pro-GRP, and TNM staging are positively correlated. By drawing the ROC curve, the results showed that the four markers combined diagnosis of lung cancer had the largest AUC, which was 0.9250 (95\% CI: 0.8866-0.9634), and the sensitivity $(93.29 \%)$ and specificity $(84.32 \%)$ were both high.

\section{Conclusions}

The levels of CEA, CYFRA21-1, SCC-Ag, and pro-GRP are of high value in the differential diagnosis of lung cancer, 
and the four joint tests have the highest diagnostic value.

\section{Acknowledgments}

Funding: The study supported by Subproject of the National Key Research and Development Program of High-end Reagents Traceable Quantity Valuation Technology and Quality Evaluation Method (No. 2019YFF0216501-L19).

\section{Footnote}

Reporting Checklist: The authors have completed the STARD reporting checklist. Available at http://dx.doi.org/10.21037/ tcr-21-527

Data Sharing Statement: Available at http://dx.doi. org/10.21037/tcr-21-527

Conflicts of Interest: All authors have completed the ICMJE uniform disclosure form (available at http://dx.doi. org/10.21037/tcr-21-527). The authors have no conflicts of interest to declare.

Ethical Statement: The authors are accountable for all aspects of the work in ensuring that questions related to the accuracy or integrity of any part of the work are appropriately investigated and resolved. The study was conducted in accordance with the Declaration of Helsinki (as revised in 2013) and was approved by Chengmai County People's Hospital (No.: 20161248). Written informed consent was obtained from the patients.

Open Access Statement: This is an Open Access article distributed in accordance with the Creative Commons Attribution-NonCommercial-NoDerivs 4.0 International License (CC BY-NC-ND 4.0), which permits the noncommercial replication and distribution of the article with the strict proviso that no changes or edits are made and the original work is properly cited (including links to both the formal publication through the relevant DOI and the license). See: https://creativecommons.org/licenses/by-nc-nd/4.0/.

\section{References}

1. Mao Y, Yang D, He J, et al. Epidemiology of Lung Cancer. Surg Oncol Clin N Am 2016;25:439-45.

2. Bade BC, Dela Cruz CS. Lung Cancer 2020:

Epidemiology, Etiology, and Prevention. Clin Chest Med
2020;41:1-24.

3. Romaszko AM, Doboszyńska A. Multiple primary lung cancer: A literature review. Adv Clin Exp Med 2018;27:725-30.

4. Zhang YX, Yuan J, Gao ZM, et al. LncRNA TUC338 promotes invasion of lung cancer by activating MAPK pathway. Eur Rev Med Pharmacol Sci 2018;22:443-9.

5. Ridge CA, McErlean AM, Ginsberg MS. Epidemiology of lung cancer. Semin Intervent Radiol 2013;30:93-8.

6. National Lung Screening Trial Research Team. Lung Cancer Incidence and Mortality with Extended Followup in the National Lung Screening Trial. J Thorac Oncol 2019;14:1732-42.

7. Hoffman RM, Atallah RP, Struble RD. Lung Cancer Screening with Low-Dose CT: a Meta-Analysis. J Gen Intern Med 2020;35:3015-25.

8. Kumar V, Becker K, Zheng HX, et al. The performance of NLST screening criteria in Asian lung cancer patients. BMC Cancer 2015;15:916.

9. Wang L, Wang D, Zheng G, et al. Clinical evaluation and therapeutic monitoring value of serum tumor markers in lung cancer. Int J Biol Markers 2016;31:e80-e87.

10. Grunnet M, Sorensen JB. Carcinoembryonic antigen (CEA) as tumor marker in lung cancer. Lung Cancer 2012;76:138-43.

11. Chen ZQ, Huang LS, Zhu B. Assessment of Seven Clinical Tumor Markers in Diagnosis of Non-Small-Cell Lung Cancer. Dis Markers 2018;2018:9845123.

12. Fu L, Wang R, Yin L, et al. CYFRA21-1 tests in the diagnosis of non-small cell lung cancer: A meta-analysis. Int J Biol Markers 2019;34:251-61.

13. Wang H, Qian J. Serum pro-gastrin-releasing peptide in diagnosis of small cell lung cancer: A meta-analysis. J Cancer Res Ther 2016;12:C260-C263.

14. Wu LX, Li XF, Chen H, et al. Combined detection of CEA and CA125 for the diagnosis for lung cancer: A metaanalysis. Cell Mol Biol (Noisy-le-grand) 2018;64:67-70.

15. Sun J, Chen X, Wang Y. Comparison of the diagnostic value of CEA combined with OPN or DKK1 in non-small cell lung cancer. Oncol Lett 2020;20:3046-52.

16. Hao C, Zhang G, Zhang L. Serum CEA levels in 49 different types of cancer and noncancer diseases. Prog Mol Biol Transl Sci 2019;162:213-27.

17. Zhou J, Diao X, Wang S. Diagnosis Value of Combined Detection of Serum SF, CEA and CRP in Non-Small Cell Lung Cancer. Cancer Manag Res 2020;12:8813-9.

18. Onyango CG, Ogonda L, Guyah B, et al. Novel biomarkers with promising benefits for diagnosis of 
cervical neoplasia: a systematic review. Infect Agent Cancer 2020;15:68.

19. Yu D, Du K, Liu T, et al. Prognostic value of tumor markers, NSE, CA125 and SCC, in operable NSCLC Patients. Int J Mol Sci 2013;14:11145-56.

20. Nakamura H, Nishimura T. History, molecular features, and clinical importance of conventional serum biomarkers in lung cancer. Surg Today 2017;47:1037-59.

21. Cavalieri S, Morelli D, Martinetti A, et al. Clinical implications for pro-GRP in small cell lung cancer. A single center experience. Int J Biol Markers 2018;33:55-61.

22. Yang Q, Zhang $\mathrm{P}, \mathrm{Wu}$ R, et al. Identifying the Best Marker

Cite this article as: Li J, Chen Y, Wang X, Wang C, Xiao M. The value of combined detection of CEA, CYFRA21-1, SCC-Ag, and pro-GRP in the differential diagnosis of lung cancer. Transl Cancer Res 2021;10(4):1900-1906. doi: 10.21037/tcr-21-527
Combination in CEA, CA125, CY211, NSE, and SCC for Lung Cancer Screening by Combining ROC Curve and Logistic Regression Analyses: Is It Feasible? Dis Markers 2018;2018:2082840.

23. Li Q, Sang S. Diagnostic Value and Clinical Significance of Combined Detection of Serum Markers CYFRA21-1, SCC Ag, NSE, CEA and ProGRP in Non-Small Cell Lung Carcinoma. Clin Lab 2020. doi: 10.7754/Clin. Lab.2020.191243.

(English Language Editor: B. Draper) 